\title{
Could the use of bedside lung ultrasound reduce the number of chest $x$-rays in the intensive care unit?
}

\author{
Etrusca Brogi ${ }^{1}$, Elena Bignami ${ }^{*}$, Anna Sidoti ${ }^{1}$, Mohammed Shawar $^{1}$, Luna Gargani ${ }^{3}$, Luigi Vetrugno ${ }^{4}$, \\ Giovanni Volpicelli ${ }^{5}$ and Francesco Forfori ${ }^{1}$
}

\begin{abstract}
Background: Lung ultrasound can be used as an alternative to chest radiography (CXR) for the diagnosis and follow-up of various lung diseases in the intensive care unit (ICU). Our aim was to evaluate the influence that introducing a routine daily use of lung ultrasound in critically ill patients may have on the number of CXRs and as a consequence, on medical costs and radiation exposure.

Methods: Data were collected by conducting a retrospective evaluation of the medical records of adult patients who needed thoracic imaging and were admitted to our academic polyvalent ICU. We compared the number of CXRs and relative costs before and after the introduction of lung ultrasound in our ICU.

Results: A total of 4134 medical records were collected from January 2010 to December 2014. We divided our population into two groups, before (Group A, 1869 patients) and after (Group B, 2265 patients) the introduction of a routine use of LUS in July 2012. Group A performed a higher number of CXRs compared to Group B (1810 vs 961, $P=0.012$ ), at an average of 0.97 vs 0.42 exams per patient. The estimated reduction of costs between Groups A and B obtained after the introduction of LUS, was 57\%. No statistically significant difference between the outcome parameters of the two groups was observed.
\end{abstract}

Conclusions: Lung ultrasound was effective in reducing the number of CXRs and relative medical costs and radiation exposure in ICU, without affecting patient outcome.

Keywords: Acute care, Ultrasound, Imaging, Ultrasound, X-Rays, Imaging, X-Rays

\section{Background}

Critically ill patients frequently need thoracic imaging due to the constant evolution of their clinical conditions. Computed tomography (CT) scans remain the gold standard imaging technique for thoracic evaluation, but transportation of patients outside the ICU is difficult and potentially harmful [1]. Chest CT scans expose patients to large doses of radiation and should be reserved for specific situations (e.g., the evaluation of mediastinal pathologies and confirmation of pulmonary embolism) [2-4]. Bedside chest X-ray (CXR) is still considered the standard of care for many diagnostic applications in the Intensive Care Unit (ICU). However, this imaging technique has important

\footnotetext{
* Correspondence: bignami.elena@hsr.it

${ }^{2}$ Department of Anesthesia and Intensive Care, IRCCS San Raffaele Scientific Institute, Via Olgettina 60, 20132 Milan, Italy

Full list of author information is available at the end of the article
}

methodological limitations and often shows low accuracy [5]. Furthermore, it is important to consider radioprotection issues. Multiple radiologic imaging exams result in an increased incidence of radiation-induced cancer in the long-term [6].

Bedside sonography has become essential in the ICU for many common applications. Particularly, lung ultrasound (LUS) has been shown to be superior to CXR as a diagnostic tool for the diagnosis of some lung conditions in critically ill patients (i.e., pneumothorax, pleural effusion, consolidation, Ventilator-Associated-Pneumonia) [7-9]. Consequently, LUS may be considered a valid alternative to CXR in some specific situations. Potentially, a systematic application of LUS may be associated with a reduced use of routine CXR and chest CT scans, without affecting patient outcome while reducing radiation exposure [10]. Also, the use of bedside LUS could 
lead to reduced medical costs, as ultrasound scanners are relatively low-cost regarding maintenance and high durability compared to other imaging modalities [11].

In our ICU the routine use of LUS was introduced in July 2012: since then, we have performed a daily LUS round in our critically ill patients. The first aim of our study was to evaluate whether the routine daily use of LUS in our ICU influenced the number of diagnostic CXRs performed in our critically ill patients. The second aim was to estimate the effective reduction of medical costs and radiation exposure that was achieved by the introduction of LUS.

\section{Methods}

This single-center, observational, retrospective cohort study took place in a university hospital in Italy from 2010 to 2014 . We retrospectively analyzed our prospectively collected database. After approval by the Research Ethics Committee of Pisa (approval number 979, 07/04/ 2016), 4134 consecutive adult patients were enrolled in the study. Written informed consent was obtained from all the patients. This study adheres to the applicable STROBE guidelines. Data extraction was performed independently by two authors (S.M., S.A.) and any discrepancy resolved prior to final analysis by discussion with a third authors (B.E.).

We recruited adult patients admitted to our ICU from January 2010 to December 2014, who needed thoracic imaging during the ordinary clinical work-up. The inclusion criteria were as follows: age $>18$ years, ability to provide written consent, and clinical indications for thoracic imaging test.

In the second half of 2012, we implemented ultrasound as the thoracic imaging technique of choice in our ICU. From then on, physicians could decide to use LUS instead of CXR for the first diagnosis, follow-up and monitoring of pleural-pulmonary conditions in the critically ill patients admitted to our ICU.

The cohort was divided into two groups using temporal criteria, as follows:

A. In Group A, we included patients admitted to the ICU from January 1, 2010 to May 31, 2012. During this period LUS was not yet implemented as a standard practice in our ICU and was only used sporadically on a consultancy basis. In this period, thoracic imaging was based on CXRs or thoracic CT scans as the standard of practice.

B. In Group B, we included patients admitted to the ICU from June 1, 2012 to December 31, 2014. In this period, thoracic ultrasound was introduced in our ICU and implemented as the standard of care for many applications: physicians could choose to rely on LUS with or without integration with radiology methods. During that period, the imaging technique of choice was LUS, then, in case of clinical doubt or technical problems, X- Rays were used to overcome the issue.

At the bedside, anterior-posterior chest radiography was performed in the supine position following standard technique using a portable X-ray unit; radiologists were responsible for reading and interpreting the digital imaging. In our ICU during the selected period (20122014), three trained critical care physicians performed LUS. The operators had acquired the level of competence defined by the American College of Chest Physicians [12]. Trained physicians had performed theoretical courses, simulated practice on manikins (at least 50 scans) and had subsequent formal supervised practice [13]. The examination consisted of a bilateral scan of the anterior and lateral chest wall with patients in the supine position. A microconvex probe was used as the first choice for LUS. Then, to help resolve cases where diagnostic doubt remains, higher frequency probes were chosen for a better visualisation of the pleural line and subpleural space. The probe was positioned longitudinally in order to visualise the "bat sign" (the pleural line and two ribs), then, placed in transversal plane. Chest wall was examined in 8 areas with one scan for each area [14]. All regions were scanned with ultrasound in order to assess pleural sliding, presence and possible number of A- and B-lines, pleural effusion, pneumothorax, lung consolidation and diaphragmatic mobility. When indicated, two dorsal scans per side were added (consolidations, aeration monitoring) [15]. Ultrasound diagnoses of lung disease were defined according to the International Consensus Conference on Lung Ultrasound [16]. LUS findings were reported on the clinical notes.

For all patients we recorded sex, age, ICU-admission diagnosis (trauma, medical, surgical patients), SAPS II score, duration of ICU stay and ICU mortality. We recorded and compared the number of CRXs performed in Group A and Group B and the costs linked to CXR prescriptions between the two groups.

The data were entered into a spreadsheet (Microsoft Excel) and analyzed using SPSS (IBM SPSS software version 21). Demographic data are shown as means and standard deviation where appropriate. Analysis of data was performed using two-tailed Mann-Whitney test or Student $\mathrm{t}$-test where appropriate: a value of P below 0.05 defined the significance. Cost for each medical exam was gathered from our regional price list.

\section{Results}

A total of 4134 medical records were identified that fulfilled our inclusion criteria: 1869 patients in Group A and 2265 
patients in Group B were included in our analysis. Demographic characteristics are shown in Table 1.

Before the introduction of a routine use of LUS, we requested 1810 chest $\mathrm{X}$-rays for diagnosis and follow-up (an average of 0.97 chest X-ray per patient) with an estimated cost of 47,060€. After the introduction of a routine use of LUS, we required 961 chest X-rays for diagnosis and follow-up (an average of 0.42 chest X-ray per patient) with an estimated cost of $24,986 €$. The significant differences in the total number of CXRs, average number per patient and estimated cost obtained before and after the introduction of routine application of LUS, are shown in Table 2. In Group B we observed a reduction in the number of CXR and relative cost by $57 \%$, in comparison to Group A (Fig. 1). No statistically significant difference was observed in SAPS II regarding duration of ICU stay and ICU mortality between the two groups.

\section{Discussion}

In this study, we evaluated the influence that introducing a routine daily use of LUS may have on the number of CXRs and relative costs, in a polyvalent ICU. We found that the implementation of daily LUS examination leads to a statistically significant reduction in the number of CXRs without affecting the outcome. The cost savings associated with the reduction in the number of CXR was also remarkable. We did not find a decrease in the number of chest $\mathrm{CT}$ scans between the groups. The overwhelming majority of our sample consisted of surgical patients requiring a brief postoperative intensive care monitoring. For short ICU stays, the implementation of LUS seems of high significance, because, conventionally, X-rays are often routinely performed just before the patients' transfer.

Regarding demographic and admission data, we decided to compare the two groups mainly on the basis of SAPS II because this score is valuable in predicting risk

Table 1 Demographics and admission data of the participants: comparison between Groups A and B

\begin{tabular}{llll}
\hline Variables & Group A & Group B & $P$ \\
\hline Age (years) & $61[14.5]$ & $63[14.1]$ & 0.79 \\
Male (\%) & 52 & 56 & 0.14 \\
Female (\%) & 48 & 44 & 0.12 \\
SAPS II & $20.2[14.5]$ & $20.6[12.3]$ & 0.28 \\
ICU mortality & 3.9 & 2.1 & \\
ICU stay & $2.74[5.72]$ & $2.85[8.06]$ & 0.36 \\
Admission diagnosis (\%): & & & \\
Trauma patient & 1 & 1 & 1 \\
Surgical patient & 86 & 89 & 0.9 \\
Medical patient & 13 & 10 & 0.9 \\
\hline
\end{tabular}

Data are presented as mean and [standard deviation] or as percentage (\%) where appropriate. SAPSII = The Simplified Acute Physiology Score II, $\mathrm{ICU}=$ Intensive care unit
Table 2 CXRs and Ct requested and relative costs: comparison between Groups A and B

\begin{tabular}{llll}
\hline & Group A & Group B & P \\
\hline Number of CXRs & 1.810 & 961 & 0.012 \\
Number of CXRs per patient & 0.97 & 0.42 & \\
Cumulative estimated cost due to CXRs $(€)$ & 47.090 & 24.986 & 0.012 \\
Number of CT & 87 & 89 & \\
\hline
\end{tabular}

Data are presented as total number. CXRs; chest X-rays

of mortality and it provides a reliable and effective estimation of the overall clinical condition of the patients at admission [17]. Consequently, we decided not to present the specific admission diagnosis and we categorized patients only on the base of three diagnoses: trauma, surgical and medical patients.

LUS was shown to be essential in our modern ICU, since it is a safe, fast bedside technique that may replace CXR for many applications. Moreover, the decreased radiation exposure represents a considerable improvement in safety and patient care. The carcinogenic effects of Xrays are extensively documented. The mechanism of radiation damage arises from the interaction between high-energy X-rays and biological material leading to radiation-induced mutations (a phenomenon known as "stochastic effect") and to radiation-induced cell death (known as "deterministic effect") [6]. Stochastic effects occur without a specific threshold dose of radiation; consequently, the risk of developing cancer from radiation exposure is essentially stochastic. Moreover, physicians have to take into account the cumulative risk of radiation exposure and that different imaging techniques require different doses of radiation. For instance, one chest CT scan has an effective radiation dose of $8 \mathrm{mSv}$, equivalent to 400 CXRs [18]. Furthermore, doctors have to consider that the risk for each dose also depends on age (higher in children, because of rapid dividing cells and life expectancy) and gender (higher in women). Consequently, the responsible use of imaging tests is vital in clinical practice. Inappropriate application of $\mathrm{X}$-ray imaging is socially and economically unjustifiable. At the bedside with critically ill patients, CXR also suffers from methodological limitations (i.e., supine anterior-posterior vs upright posterior-anterior views, non-collaborating patient vs patient controlling his breath, possibility of adjunctive lateral views). These aspects, along with the presence of other concomitant lung diseases and complexity of critically ill patients, contributes to poor-quality CXR imaging and the possibility of wrong interpretations [19].

In this scenario, bedside point-of-care LUS has led to important changes in clinical practice, as it allows clinicians to rapidly answer specific questions, to guide therapy and to assess the efficacy of ongoing interventions, 

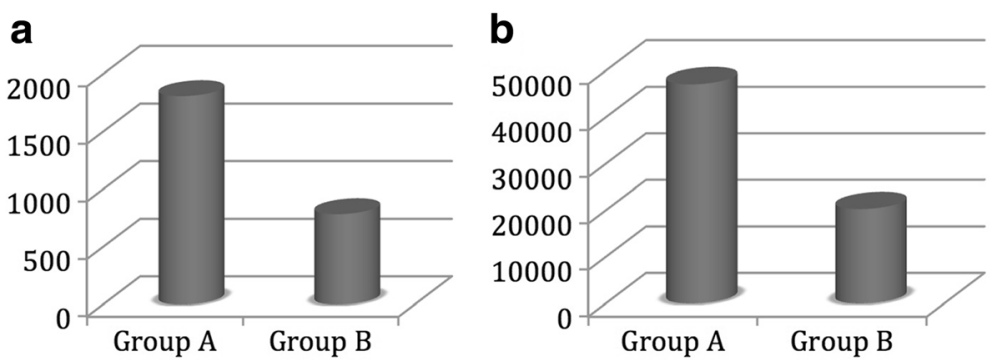

Fig. 1 Clustered cylinder chart illustrates the differences in number ( $\mathbf{a}$, left chart) and cost (b, right chart) of chest $X$-rays between Group A and Group B. The $Y$-axis represents total number of chest $X$-rays ( $A$, left chart) or the total cost in Euros (B, right chart)

with high diagnostic accuracy and specificity. In 2004 Lichtenstein et al. evaluated the diagnostic accuracy of auscultation, bedside CXR and LUS in comparison to thoracic CT in patients with ARDS. These Authors showed that bedside CXR and physical examination had a lower sensitivity, specificity and diagnostic accuracy than LUS in the diagnosis of some lung conditions (i.e., pleural effusions, interstitial syndrome, alveolar consolidation) [5]. With the progression of literature on the topic and affirmation of a concept that has recently become evidence-based, the use of point-of-care ultrasound in the ICU has increased dramatically. Lee et al. [20] conducted a retrospective study in order to estimate the expected change over years in the use of imaging tests. They found a decrease in second-level imaging tests (CT by $21 \%$ and MRI by $6 \%$ ) together with an increase in the use of ultrasound by $18.9 \%$, and as a direct consequence a decrease in medical charges, without affecting patient outcome. In 2015, Zieleskiewicz et al. [11] investigated retrospectively the effect of ultrasound chest imaging on the reduction of CXRs and medical costs. They observed a statistically significant decrease in the number of CXRs due to the introduction of ultrasound in their ICU, without affecting mortality. Indeed, they did not find a decrease in the number of chest CT scans. Furthermore, Peris et al. [21] published an observational control study on 376 intensive care patients. The patient cohort was divided into two groups using temporal criteria (before and after the introduction of routine LUS). The data showed a statistically significant decrease in the number of CXRs and CT scans performed after the implementation of LUS. The conclusion of these Authors was that LUS may be used as an alternative to thoracic radiology [8] without affecting patient outcome [11]. Moreover, LUS is a valuable tool for selecting patients who effectively need a more advanced imaging technology (i.e., CT scan, MRI), thus contributing to reducing the number of inappropriate ionizing imaging tests.

The well-known and repeatedly cited limitation of sonography is operator dependency. A correct ultrasonography examination is directly related to operator skill, training, and experience. However, a combination of increased medical knowledge, ultrasound proficiency and appropriate training of operators could reduce errors [12]. Moreover, LUS is mainly based on simple basic signs and in many studies was shown to be highly reproducible in the hands of operators with different skills and experience, although the importance of correct training is always emphasized. Definitely, the benefits of implementing LUS in the ICU usually outweigh the pitfalls, if sonography is performed by trained physicians [22].

Our study presents several limitations. First of all, due to the retrospective nature of the study, it was not possible to gather information about the clinical indications for performing an imaging technique or when CXR was requested on the basis of US findings. For the same reason, it was not possible to evaluate how many chest $\mathrm{x}$-rays or LUS examinations were normal and how many were pathological in the two groups. Second, the use of CXR or US was at the physician's discretion, which may vary depending on the individual's sensitivity and consideration. Since LUS is a relative novelty and is still considered a possible alternative, it was not feasible to set up a standard protocol for comparison. For these reasons, the reader should consider that the measured decrease in the number of CXRs observed in our study may not necessarily correspond to other situations. A future study on the subject should be performed, applying fixed protocols based on the evidence-based superiority of LUS in some applications. Third, it was not possible to collect information on overall mortality but only on ICU mortality, as patients were not followed-up after discharge from the ICU.

\section{Conclusions}

In conclusion, this study shows the importance and effectiveness of LUS in reducing the number of CXRs performed in an academic polyvalent ICU. Routine LUS application, even when only left to the discretion of the caring physician, allows decreasing the use of ionizing procedures as well as related biological and economic costs. 


\section{Abbreviations}

CT: Computed tomography; CXR: Chest radiography; ICU: intensive care unit: LUS: Lung ultrasound; MRI: Magnetic resonance imaging; SAPS II: Simplified Acute Physiology Score

\section{Acknowledgements}

Not applicable.

\section{Funding}

No external funding declared.

\section{Availability of data and materials}

Are available from the corresponding author on reasonable request.

\section{Authors' contributions}

EB (first author) acquisition of data, analysis and interpretation of data, drafting/revising the manuscript, control and guarantee that all aspects of the work was investigated and resolved. EB approved the final manuscript. EB (corresponding author) acquisition of data, analysis and interpretation of data, drafting/revising the manuscript, control and guarantee that all aspects of the work was investigated and resolved. EB approved the final manuscript. AS acquisition of data, analysis and interpretation of data, drafting/revising the manuscript, control and guarantee that all aspects of the work was investigated and resolved. AS approved the final manuscript. MS acquisition of data, analysis and interpretation of data, drafting/revising the manuscript, control and guarantee that all aspects of the work was investigated and resolved. MS approved the final manuscript. LG acquisition of data, analysis and interpretation of data, drafting/revising the manuscript, control and guarantee that all aspects of the work was investigated and resolved. LG approved the final manuscript. LV acquisition of data, analysis and interpretation of data, drafting/revising the manuscript, control and guarantee that all aspects of the work was investigated and resolved. LV approved the final manuscript. GV acquisition of data, analysis and interpretation of data, drafting/revising the manuscript, control and guarantee that all aspects of the work was investigated and resolved. GV approved the final manuscript. FF study concept and design, acquisition of data, analysis or interpretation of data, drafting/revising the manuscript, control and guarantee that all aspects of the work was investigated and resolved, critical revision of the manuscript for important intellectual content, study supervision. FF approved the final manuscript.

\section{Ethics approval and consent to participate}

Ethics approval was obtained by the Research Ethics Committee of Pisa (approval number 979, 07/04/2016). Written informed consent was obtained from all the patients.

\section{Consent for publication}

Not applicable.

\section{Competing interests}

Etrusca Brogi has no conflict of interest to declare. Elena Bignami has no conflict of interest to declare. Anna Sidoti has no conflict of interest to declare. Mohammed Shawar has no conflict of interest to declare. Luna Gargani received a speaker's honorarium from GE Healthcare. Luigi Vetrugno has no conflict of interest to declare. Giovanni Volpicelli has no conflict of interest to declare. Francesco Forfori has no conflict of interest to declare.

\section{Publisher's Note}

Springer Nature remains neutral with regard to jurisdictional claims in published maps and institutional affiliations.

\section{Author details}

'Department of Anesthesia and Intensive Care, University of Pisa, Pisa, Italy. ${ }^{2}$ Department of Anesthesia and Intensive Care, IRCCS San Raffaele Scientific Institute, Via Olgettina 60, 20132 Milan, Italy. ${ }^{3}$ Institute of Clinical Physiology National Research Council, Pisa, Italy. ${ }^{4}$ Department of Medicine, University of Udine, Udine, Italy. ${ }^{5}$ Department of Emergency Medicine, San Luigi Gonzaga University Hospital, Orbassano, Torino, Italy.
Received: 23 May 2017 Accepted: 5 September 2017

Published online: 13 September 2017

\section{References}

1. Dunn MJ, Gwinnutt CL, Gray AJ. Critical care in the emergency department: patient transfer. Emerg Med J. 2007;24(1):40-4.

2. Tecce PM, Fishman EK, Kuhlman JE. CT evaluation of the anterior mediastinum: spectrum of disease. Radiographics. 1994;14(5):973-90.

3. Brenner DJ, Hall EJ. Computed tomography-an increasing source of radiation exposure. N Engl J Med. 2007;357(22):2277-84.

4. Tapson VF. Advances in the diagnosis and treatment of acute pulmonary embolism. F1000 Med Rep. 2012;4:9. doi:10.3410/M4-9.

5. Lichtenstein D, Goldstein I, Mourgeon E, Cluzel P, Grenier P, Rouby JJ. Comparative diagnostic performances of auscultation, chest radiography, and lung ultrasonography in acute respiratory distress syndrome. Anesthesiology. 2004;100(1):9-15.

6. Gargani L, Picano E. The risk of cumulative radiation exposure in chest imaging and the advantage of bedside ultrasound. Crit Ultrasound J. 2015;7:4.

7. Agricola E, Arbelot C, Blaivas M, Bouhemad B, Copetti R, Dean A, Dulchavsky S, Elbarbary M, Gargani L, Hoppmann R, et al. Ultrasound performs better than radiographs. Thorax. 2011;66(9):828-9. author reply 829

8. Xirouchaki N, Magkanas E, Vaporidi K, Kondili E, Plataki M, Patrianakos A, Akoumianaki E, Georgopoulos D. Lung ultrasound in critically ill patients: comparison with bedside chest radiography. Intensive Care Med. 2011;37(9): 1488-93.

9. Mongodi S, Via G, Girard M, Rouquette I, Misset B, Braschi A, Mojoli F, Bouhemad B. Lung Ultrasound for Early Diagnosis of Ventilator-Associated Pneumonia. Chest. 2016;149(4):969-80.

10. Biasucci DG, Cutuli SL, Pizza A, Cavaliere F. Clinically integrated ultrasound for decreasing the number of chest $\mathrm{x}$-rays in the intensive care unit: It is high time to move forward a "global" use of ultrasound. J Crit Care. 2015;30(5):1137-8

11. Zieleskiewicz L, Cornesse A, Hammad E, Haddam M, Brun C, Vigne C, Meyssignac B, Remacle A, Chaumoitre K, Antonini F, et al. Implementation of lung ultrasound in polyvalent intensive care unit: Impact on irradiation and medical cost. Anaesth Crit Care Pain Med. 2015;34(1):41-4.

12. Mayo PH, Beaulieu Y, Doelken P, Feller-Kopman D, Harrod C, Kaplan A, Oropello J, Vieillard-Baron A, Axler O, Lichtenstein D, et al. American College of Chest Physicians/La Societe de Reanimation de Lanque Francaise statement on competence in critical care ultrasonography. Chest. 2009;135(4):1050-60.

13. Salamonsen M, McGrath D, Steiler G, Ware R, Colt H, Fielding D. A new instrument to assess physician skill at thoracic ultrasound, including pleural effusion markup. Chest. 2013;144(3):930-4

14. Volpicelli G, Mussa A, Garofalo G, Cardinale L, Casoli G, Perotto F, Fava C, Frascisco M. Bedside lung ultrasound in the assessment of alveolarinterstitial syndrome. Am J Emerg Med. 2006;24(6):689-96.

15. Gargani L, Volpicelli G. How I do it: lung ultrasound. Cardiov Ultrasound. 2014;12:25.

16. Volpicelli G, Elbarbary M, Blaivas M, Lichtenstein DA, Mathis G, Kirkpatrick AW, Melniker L, Gargani L, Noble VE, Via G, et al. International evidencebased recommendations for point-of-care lung ultrasound. Intensive Care Med. 2012;38(4):577-91.

17. Allyn J, Ferdynus C, Bohrer M, Dalban C, Valance D, Allou N. Simplified Acute Physiology Score II as Predictor of Mortality in Intensive Care Units: A Decision Curve Analysis. PLoS ONE. 2016;11(10).

18. Picano E. Sustainability of medical imaging. BMJ. 2004;328(7439):578-80.

19. Basi SK, Marrie TJ, Huang JQ, Majumdar SR. Patients admitted to hospital with suspected pneumonia and normal chest radiographs: epidemiology, microbiology, and outcomes. Am J Med. 2004;117(5):305-11.

20. Lee J, Geyer B, Naraghi L, Kaafarani HM, Eikermann M, Yeh DD, Bajwa EK, Cobb JP, Raja AS. Advanced imaging use in intensive care units has decreased, resulting in lower charges without negative effects on patient outcomes. J Crit Care. 2015;30(3):460-4.

21. Peris A, Tutino L, Zagli G, Batacchi S, Cianchi G, Spina R, Bonizzoli M, Migliaccio L, Perretta L, Bartolini M, et al. The use of point-of-care bedside lung ultrasound significantly reduces the number of radiographs and computed tomography scans in critically ill patients. Anesth Analg. 2010; 111(3):687-92.

22. Pinto A, Pinto F, Faggian A, Rubini G, Caranci F, Macarini L, Genovese EA, Brunese L. Sources of error in emergency ultrasonography. Crit Ultrasound J. 2013;5 Suppl 1:S1. 